\title{
Ge/GaAs Based Negative Capacitance Tunnel FET Biosensor: Proposal and Sensitivity Analysis
}

\author{
Omdarshan Paul ${ }^{1} \cdot$ Chithraja Rajan $^{1} \cdot$ Dip Prakash Samajdar ${ }^{1} \cdot$ Tarek Hidouri $^{2} \cdot$ Samia Nasr $^{3}$
}

Received: 30 November 2021 / Accepted: 20 February 2022 / Published online: 7 March 2022

(C) The Author(s), under exclusive licence to Springer Nature B.V. 2022

\begin{abstract}
A highly sensitive, accurate, fast and power efficient biosensor is the need of the hour. Undoubtedly, dielectrically modulated (DM) tunnel FET (TFET) assures better sensitivity as compared to MOSFET biosensors in case of label-free biosensing. However, there exists immense possibilities to upgrade TFET biosensor properties through the improvement of its DC characteristics. Therefore, in this paper a ferroelectric (FE) gate oxide and a hetero material (HM) source/drain-channel based TFET is designed for biosensor applications. A FE layer of $\mathrm{HfZrO}_{2}$ above $\mathrm{SiO}_{2}$ gives rise to negative capacitance (NC) effect that causes voltage amplification and hence, boosts subthreshold swing (SS) and $\mathrm{I}_{\mathrm{ON}} / \mathrm{I}_{\mathrm{OFF}}$ ratio. In addition, use of a low band gap material $(\mathrm{Ge})$ in source and a high band gap material $(\mathrm{GaAs})$ in drain-channel junctions enhances the probability of band-to-band-tunneling (BTBT) of charge carriers. Further, to introduce biomolecules, a cavity is impinged below $\mathrm{HfZrO}_{2}$ near $\mathrm{SiO}_{2}$ above source/channel junction that modulates BTBT as a function of charge density $\left(\mathrm{N}_{\mathrm{f}}\right)$ and dielectric constant $(\mathrm{K})$. This paper presents a detailed comparative analysis of Ge/GaAs-NCTFET and Ge/GaAs-TFET biosensors for different $\mathrm{K}_{\text {and }} \mathrm{N}_{\mathrm{f}}$ values from which we can conclude that the incorporation of NC effect in TFET biosensors leads to enhanced sensitivity with high speed and low power consumption.
\end{abstract}

Keywords Negative capacitance $\cdot$ Ferroelectric $\cdot$ Band-to-band tunneling $(B T B T) \cdot$ Potential intensification ·

Dielectric modulated $\cdot$ Hetero material $\cdot$ Biosensor $\cdot$ Tunnel FET

\section{Introduction}

In December 2019, serious respiratory misery with pneumonia-like symptoms was accounted all over the world. Later, the genomic investigations revealed that the infection shares a nearby hereditary likeliness to the SARS (Severe Acute Respiratory Syndrome) like COVID-19 [1]. Consequently, in order to check the rapid infection transmission rate, accurate analysis of COVID-19 is required through

Dip Prakash Samajdar

dipprakash010@gmail.com

Omdarshan Paul

1912303@iiitdmj.ac.in

Chithraja Rajan

rajan.chithraja@gmail.com

Tarek Hidouri

hidouritarek@gmail.com

Samia Nasr

smohmed@kku.edu.sa systematic testing of the swab specimen of the infected individuals [2]. Testing individuals for any popular contamination includes detection of biomolecules in the collected specimens in the presence of some analytes such as viral proteins, flawless viral particles, nucleic acids (RNA and DNA) and antibodies created by the patient's immunoresponse [2-6]. Thus, broadening the range of sensitivity and selectivity of portable biosensors with enhanced lifetime, effectiveness and robustness is the need of the present age.

1 Micro and Nano Computational Lab, Electronics and Communication Engineering Discipline, PDPM Indian Institute of Information Technology, Design and Manufacturing, Jabalpur, Madhya Pradesh, India

2 Micro-Optoelectronic and Nanostructures Laboratory, LR99ES29, Department of Physics, Faculty of Sciences Monastir, University of Monastir, Street of Environment, 5019 Monastir, Tunisia

3 Advanced Functional Materials \& Optoelectronic Laboratory (AFMOL), Department of Physics, Faculty of Science, King Khalid University, P.O. Box 9004, Abha, Saudi Arabia 
FET [7-12] based biosensors are generally utilized because of their possibility to detect organic species. Nonetheless, they experience some negative effects during the detection of neutral biomolecules. Label free FET [13-17] biosensors utilizes either chemical or biological receptors to recognize biomolecules in a specimen and provide limited data on affinity, and selectivity. Apart from this, the scaling of bulk MOSFET for miniaturization and fabrication of compact biosensors brings in some unavoidable limits: identification time is high because of $\frac{k T}{q}$ tyranny [SS $>60 \mathrm{mV} /$ decade], drain-induced barrier lowering (DIBL), high power utilization because of leakage current, low $\mathrm{I}_{\mathrm{ON}} / \mathrm{I}_{\mathrm{OFF}}$ ratio, and supply voltage scaling limit $[18,19]$. For these inherent shortcomings of FET-based sensors, a TFET based biosensor is widely investigated as a possible alternative to FET because of its capacity to give better sensitivity, improved reaction time, energy-efficient because of low leakage current, and its capability to overcome almost all previously mentioned issues associated with FETs [20-22]. However, creating abrupt junction in TFETs is a hectic task because of charge diffusion from source to channel [23]. Hence, it suffers from random dopant fluctuations (RDF), which degrades the device performance. Nevertheless, TFET endures low ON current $\left(\mathrm{I}_{\mathrm{ON}}\right)$ and higher ambipolarity, which restricts its utilization in real time applications. As a result, sensitivity of TFET based biosensor is comparatively low because of low $\mathrm{I}_{\mathrm{ON}}$. In spite of several attempts to improve the sensitivity of TFET based biosensors through structural modifications, negligible progress has been made in the field of fabrication of complex TFET device architectures and their actual implementation within the scope of contemporary CMOS fabrication techniques. However, the need of faster and accurate biosensors without adding extra burden to expensive manufacturing units is the main motivation for exploring the advantages of negative capacitance (NC) based hetero material TFET for biosensing applications in this manuscript.

Fundamentally, NC arises out of ferroelectric (FE) [24] material property and is first utilized in MOSFET to reduce OFF current $\left(\mathrm{I}_{\mathrm{OFF}}\right)$ and SS [25]. At the point when an outer potential is applied across a FE material, the dipole moment causes polarization, thereby leading to tunable NC. Hence, any FE material above oxide layer in a FET counteracts effective gate capacitance because of capacitance matching which prompts voltage intensification [26]. This gives impressive improvement in $\mathrm{I}_{\mathrm{ON}}, \mathrm{I}_{\mathrm{OFF}}$ and $\mathrm{SS}$ in NC-MOSFET. Although, NC-MOSFET actually consumes more power as compared to conventional TFET due to high $\mathrm{I}_{\mathrm{OFF}}$ compared to TFET, it delivers more $\mathrm{I}_{\mathrm{ON}}$ than traditional MOSFET. Therefore, NC- TFET can be used to detect biomolecules with better sensitivity because of its high $\mathrm{I}_{\mathrm{ON}}$ and better SS as compared to conventional TFET and lower power dissipation due to low $\mathrm{I}_{\mathrm{OFF}}$. However, it is evident from the literature review that Group III-V and IV semiconductors drastically improves TFET electrical characteristics as compared to Si-TFET [27]. Different Group III-V and IV semiconductor materials (InAs/Si, $\mathrm{SiGe} / \mathrm{Si}$ ) are examined to improve TFET attributes and it is found that $\mathrm{Ge} / \mathrm{GaAs}$ based TFET provides exceptional $\mathrm{I}_{\mathrm{ON}}$ with low ambipolarity. Therefore, in this manuscript, rather than utilizing $\mathrm{Si}$ in the body, GaAs and Ge are employed in drain-channel and source of NC-TFET respectively. This increases the slope of the energy bands because of the lower energy band gap at source side than $\mathrm{Si}$, which results in better tunneling of charge carriers from source to channel and FE-DE gate stack provides further voltage amplification at the surface. Moreover, to collect biomolecules, a nano cavity is made inside the gate dielectric stack. Consequently, Ge/GaAs-NC-TFET has been investigated in terms of sensitivity, drain current $\left(I_{d}\right)$, energy band diagram and SS and the results are compared with the Ge/ GaAs-TFET based biosensor. However, the fundamental focus in this manuscript is to study the impact of the variation of charge density and dielectric constant on the DC characteristics of the proposed biosensor.

\section{Device Description and Calibration}

A cross-sectional view of $\mathrm{Ge} / \mathrm{GaAs}$ TFET biosensor and $\mathrm{NC}-\mathrm{Ge} / \mathrm{GaAs}-\mathrm{TFET}$ based biosensor with a FE layer of $\mathrm{HfZrO}_{2}$ and $\mathrm{SiO}_{2}$ as an interface layer is illustrated in Fig. 1. The channel is surrounded by double gate architecture. Silvaco ATLAS TCAD [28] tool is utilized to acquire simulation results of our device and models are calibrated with the experimental FET and NC-FET [29] as demonstrated in Fig. 2a and TFET [30] in Fig. 2b. Simulated results show close resemblance with the experimental data to confirm our model validity. The calibration procedure is almost similar as adopted in [27] for simulation, nonlocal band-to-band tunnelling (BTBT) model is used, which computes the speed of tunnelling at both the tunnelling junctions. By utilizing Shockley-Read-Hall (SRH) recombination and Auger model, the impact of leakage current and mobility are estimated. Likewise, the Fermi-Dirac carrier statistics model and the doping-dependent bandgap narrowing (BGN) model are used. Additionally, to take into account the FE properties, Ferro model is used in our mathematical computation. The physical dimensions of TFET Biosensor and NCTFET Biosensor are listed in Table 1.

\section{Results and Discussion}

The biomolecules are charged or uncharged biological entities present in cells and living organisms. While the simulation of 
Fig. 1 Cross sectional view of a Ge/GaAs TFET biosensor, b Ge/ GaAs NCTFET biosensor

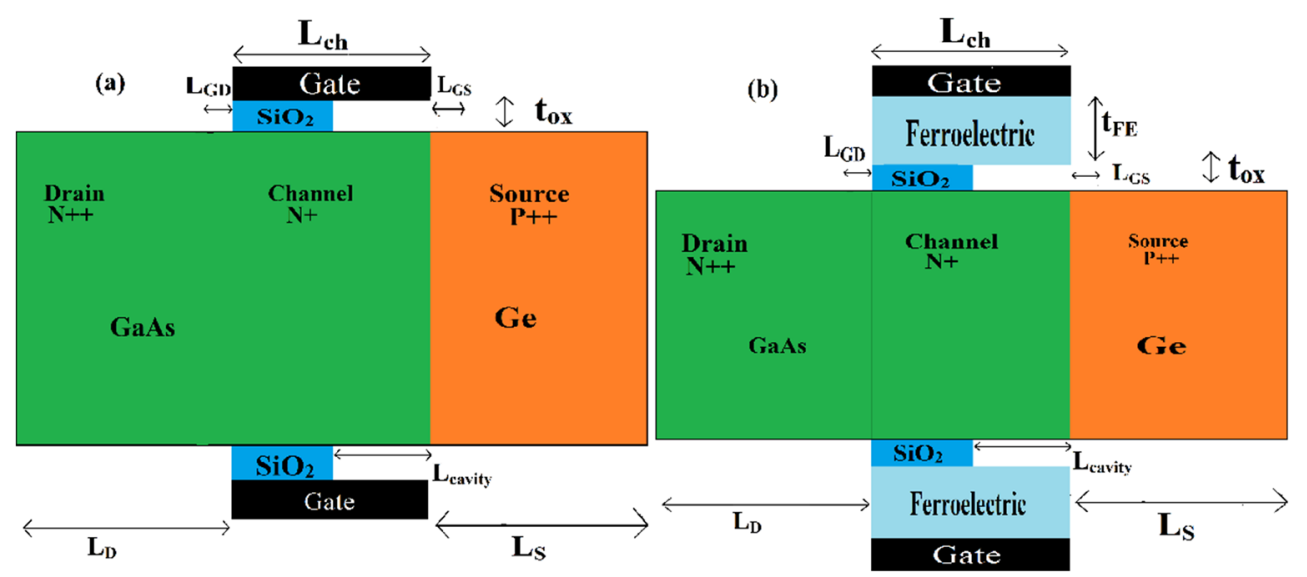

uncharged biomolecules takes into consideration the dielectric constant $(\mathrm{K})$ of the biomolecules, the charged biomolecules are simulated based on both the charge density $\left(\mathrm{N}_{\mathrm{f}}\right)$ and dielectric constant $(\mathrm{K})$ of charged biomolecules. The presence of charged or neutral biomolecules in the cavity between the semiconductor-oxide interface and gate-ferroelectric stack modulates the dielectric constant in the cavity region, which in turn affects the Effective Oxide Thickness (EOT) and the electrical parameters such as electric field, surface potential, etc. In this manuscript, we have considered a variety of well-known biomolecules with different dielectric constants. We have also taken into consideration positive and negative values of charge densities of the charged biomolecules (= $-1 \times 10^{10},-1 \times 10^{-11},-5 \times 10^{-11},-1 \times 10^{-12}, 1 \times 10^{10}, 1 \times 10^{11}$, $5 \times 10^{11}, 1 \times 10^{12}$ ) for simulation purposes and to study the impact of charge variation on the electrical characteristics of the proposed biosensor. For example, single strand of non-hybridized DNA with predefined values of dielectric constant and charge is considered to be trapped in the cavity area to obtain sensitivity of Ge/GaAs-TFET and Ge/ GaAs-NC-TFET biosensor. GaAs and Ge are utilized in drain-channel and source respectively, which expands the gradient of the energy bands because of the lower energy band gap at source side in comparison to that of Si. FE-DE gate stack above the channel region is used for potential amplification at the surface.

Figures 3, 4, 5 present the energy band diagram (EBD) of $\mathrm{Ge} / \mathrm{GaAs}$-TFET and Ge/GaAs-NCTFET by considering neutral and charged biomolecules. In (Fig. 3a, b), the abruptness of the energy band increases with an increment in the $\mathrm{K}$ value of biomolecules. Note that as gate voltage is applied, charge carriers are able to tunnel from the valence band of the channel to the conduction band of source in $\mathrm{Ge} /$ GaAs-TFET and Ge/GaAs-NCTFET based biosensors. Under ON condition, tunneling of charge carriers and intensification of surface potential is more for $\mathrm{Ge} /$ GaAs-NCTFET biosensor because of the FE layer, as compared to Ge/GaAs-TFET biosensor.

Figure $4 a, b$ represent the effect of positively charged biomolecules on EBD. Existence of positively charged analytes within cavity prompts increase (decrease) in tunneling width between drain/channel (source/channel) intersections for negative (positive) gate voltage. Therefore, Ge/GaAs-NCTFET biosensor with positively charged biomolecules in the cavity has better band bending unlike Ge/GaAs-TFET biosensor. The effect of negatively charged biomolecules on energy band is portrayed in (Fig. 5a, b). With the increase of negative charge density, hole formation in the channel region rises and consequently, for the $\mathrm{ON}$ state band bending decreases.
Fig. 2 Calibration of a FET and NCFET [29] b TFET [30]
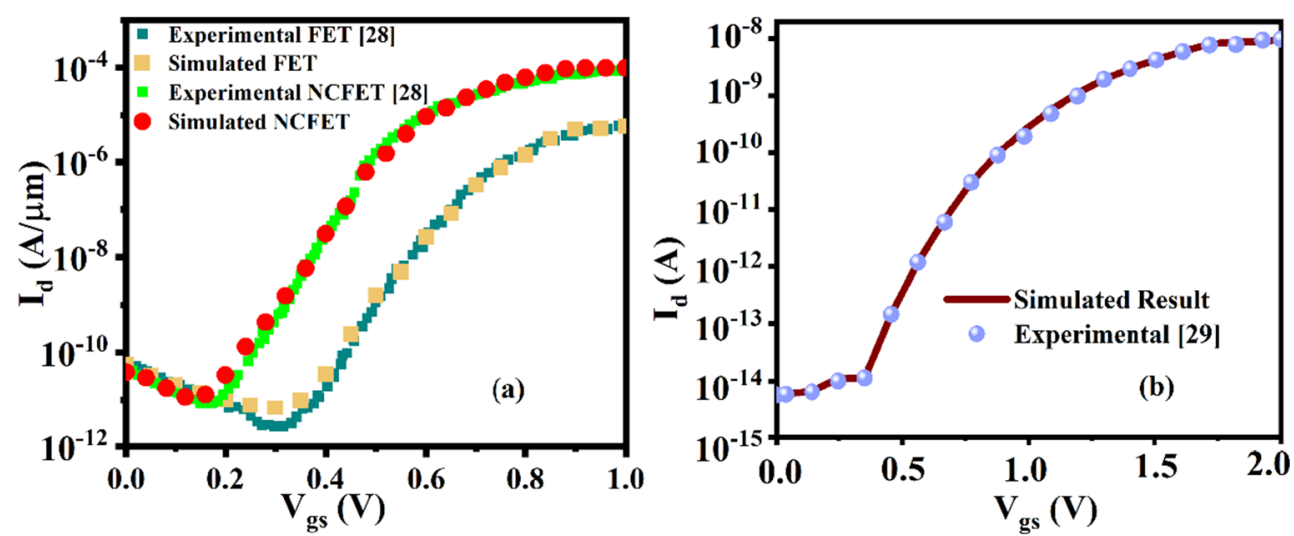
Table 1 Dimensions of TFET biosensor and NCTFET biosensor
Fig. 3 EBD in ON state of neutral biomolecules for a $\mathrm{Ge} / \mathrm{GaAs}$ TFET biosensor, b Ge/GaAs NCTFET biosensor

\begin{tabular}{|c|c|c|}
\hline Parameters & TFET-biosensor & NCTFET-biosensor \\
\hline Drain Length $\left(\mathrm{L}_{\mathrm{D}}\right)$ & $20 \mathrm{~nm}$ & $20 \mathrm{~nm}$ \\
\hline Source Length $\left(\mathrm{L}_{\mathrm{S}}\right)$ & $20 \mathrm{~nm}$ & $20 \mathrm{~nm}$ \\
\hline Gate Length $\left(\mathrm{L}_{\mathrm{G}}\right)$ & $20 \mathrm{~nm}$ & $20 \mathrm{~nm}$ \\
\hline Gate to Source spacer Length $\left(\mathrm{L}_{\mathrm{GS}}\right)$ & $5 \mathrm{~nm}$ & $5 \mathrm{~nm}$ \\
\hline Gate to Drain spacer Length $\left(\mathrm{L}_{\mathrm{GD}}\right)$ & $5 \mathrm{~nm}$ & $5 \mathrm{~nm}$ \\
\hline Source Doping $(\mathrm{P}++)$ & $10^{20} \mathrm{~cm}^{-3}$ & $10^{20} \mathrm{~cm}^{-3}$ \\
\hline Drain Doping $(\mathrm{N}++)$ & $10^{18} \mathrm{~cm}^{-3}$ & $10^{18} \mathrm{~cm}^{-3}$ \\
\hline Channel Doping $(\mathrm{N}+)$ & $10^{17} \mathrm{~cm}^{-3}$ & $10^{17} \mathrm{~cm}^{-3}$ \\
\hline Work function of Gate & $4.2 \mathrm{eV}$ & $4.2 \mathrm{eV}$ \\
\hline Oxide thickness ( $\left.t_{\mathrm{OX}}\right)$ & $2 \mathrm{~nm}$ & $2 \mathrm{~nm}$ \\
\hline FE thickness $\left(t_{\mathrm{FE}}\right)$ & - & $5 \mathrm{~nm}$ \\
\hline Coercive field $\left(\mathrm{E}_{\mathrm{C}}\right)$ & - & $1.2 \mathrm{MV} / \mathrm{cm}[31]$ \\
\hline Remnant Polarization $\left(\mathrm{P}_{\mathrm{r}}\right)$ & - & $15 \mu \mathrm{C} / \mathrm{cm}^{2}[32]$ \\
\hline
\end{tabular}
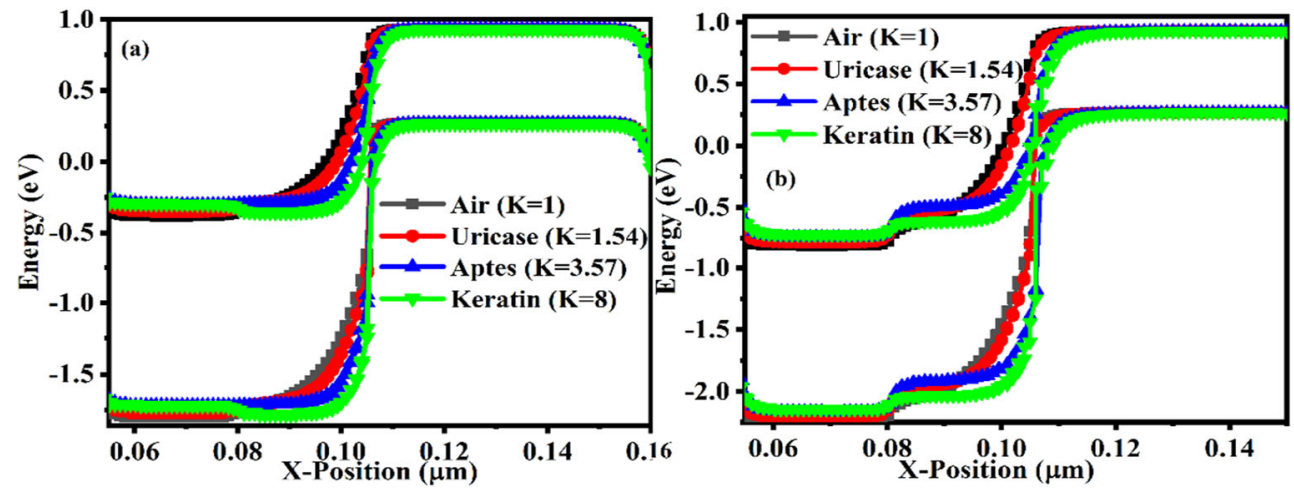

It can be seen from the plot that Ge/GaAs-NCTFET biosensor exhibits better band bending as compared to Ge/GaAs-TFET biosensor in case of negatively charged analytes in the cavity.

Figure $6 \mathrm{a}, \mathrm{b}$ shows impact of neutral biomolecules of both Ge/GaAs-TFET and Ge/GaAs-NCTFET based biosensors on transfer characteristics. It can be observed from the figures that $\mathrm{I}_{\mathrm{ON}}$ increases with the increment in $\mathrm{K}$ of the biomolecules. A maximum $\mathrm{I}_{\mathrm{ON}}$ of $9.56 \times 10^{-5} \mathrm{~A} / \mu \mathrm{m}$ in Ge/GaAs-NCTFET and $8.34 \times 10^{-6}$ in Ge/GaAs-TFET is obtained for $\mathrm{K}=12$. The capacitance enhancement can also be noticed as $\mathrm{K}$ value of neutral biomolecules increases.

Accordingly, the band bending increases at the drain/ channel and source/channel interface, with the capacitance increasing during positive $\mathrm{V}_{\mathrm{gs}}$. As capacitance is directly proportional to drain current, it results in driving current enhancement with increasing $\mathrm{K}$ value. High value of current in $\mathrm{Ge} /$
Fig. 4 EBD in ON state for positively charged biomolecules of $\mathbf{a} \mathrm{Ge} / \mathrm{GaAs}$ TFET biosensor, $\mathbf{b}$ $\mathrm{Ge} / \mathrm{GaAs}$ NCTFET biosensor
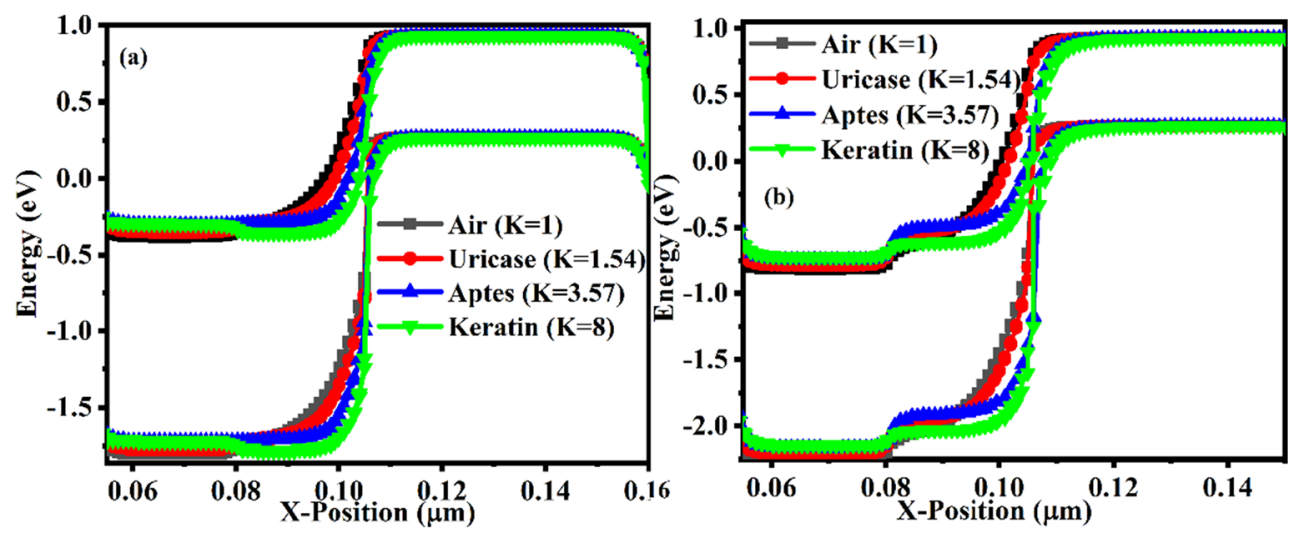
Fig. $5 \mathrm{EBD}$ in $\mathrm{ON}$ state for negatively charged biomolecules of $\mathbf{a} \mathrm{Ge} / \mathrm{GaAs}$ TFET biosensor, $\mathbf{b}$ $\mathrm{Ge} / \mathrm{GaAs}$ NC-TFET biosensor
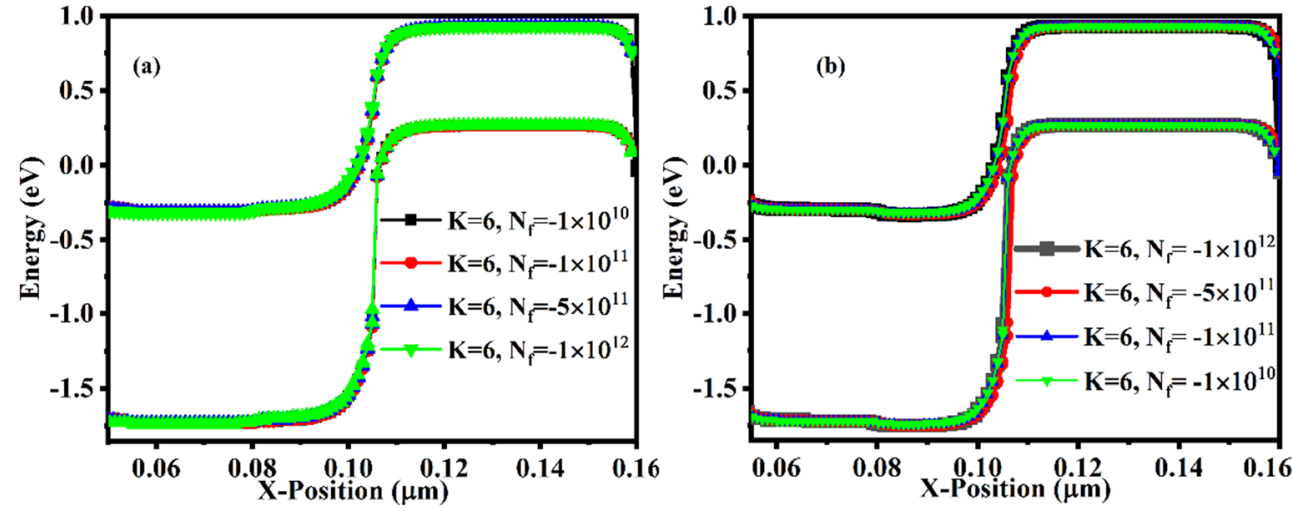

Fig. $6 I_{d} \sim V_{g s}$ characteristics of neutral biomolecules for a $\mathrm{Ge} /$ GaAs TFET biosensor b Ge/ GaAs NC-TFET biosensor
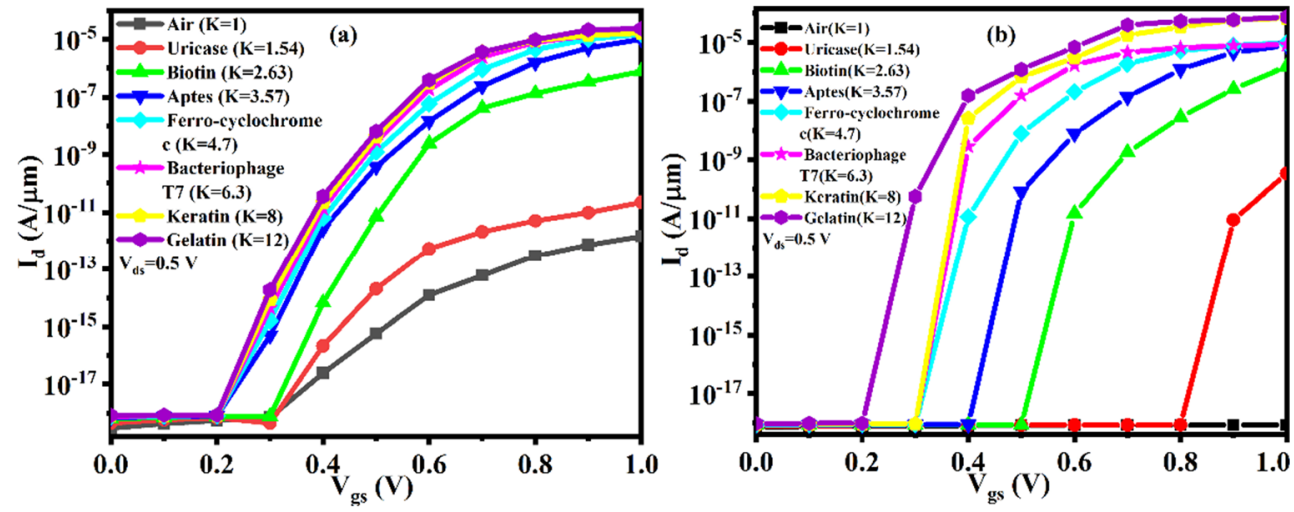

GaAs-NCTFET biosensor as compared to Ge/GaAs TFET biosensor is due to voltage intensification at FE-DE interface. In channel area, electron concentration increases as immobile positively charged biomolecules accumulates in the cavity. With the decrease in barrier height between the valence band and conduction band of source and channel respectively due to the increase in interface charges, the channel current increases. Thus, there is an increase in $\mathrm{I}_{\mathrm{ON}}$, which can be perceived from characteristics of Ge/GaAs-TFET and Ge/ GaAs-NCTFET in Fig. 7a, b.

Figure $8 \mathrm{a}, \mathrm{b}$ depict the plot of drain current of Ge/ GaAs-TFET and Ge/GaAs-NCTFET biosensors in the presence of negatively charged biomolecules in the cavity. Note that the drain current produced for negatively charged particles is comparatively less than that of the positively charged biomolecules. Though there is a rise in drain current with an accumulation of the interfacing charges of the biomolecules, same $\mathrm{I}_{\mathrm{OFF}}$ is maintained irrespective of $\mathrm{I}_{\mathrm{ON}}$. Therefore, from the transfer characteristics of Ge/GaAs-NC-TFET biosensor, we observed that drain current improves as compared to $\mathrm{Ge} /$ GaAs-TFET based biosensor. In general, the design of $\mathrm{Ge} /$ GaAs-NC-TFET is based on capacitance matching condition i.e. $\left|C_{\mathrm{FE}}\right|=\mathrm{C}_{\mathrm{TFET}}$ for voltage amplification.

Figure $9 \mathrm{a}, \mathrm{b}$ portray the transit time $(\tau)$ of both the biosensors. $\tau$ of a device should be low for quicker activity for effective analog and digital applications [33]. It is obvious
Fig. $7 \mathrm{I}_{\mathrm{d}} \sim \mathrm{V}_{\mathrm{gs}}$ characteristics of positively charged biomolecules for a Ge/GaAs TFET biosensor b Ge/GaAs NCTFET biosensor
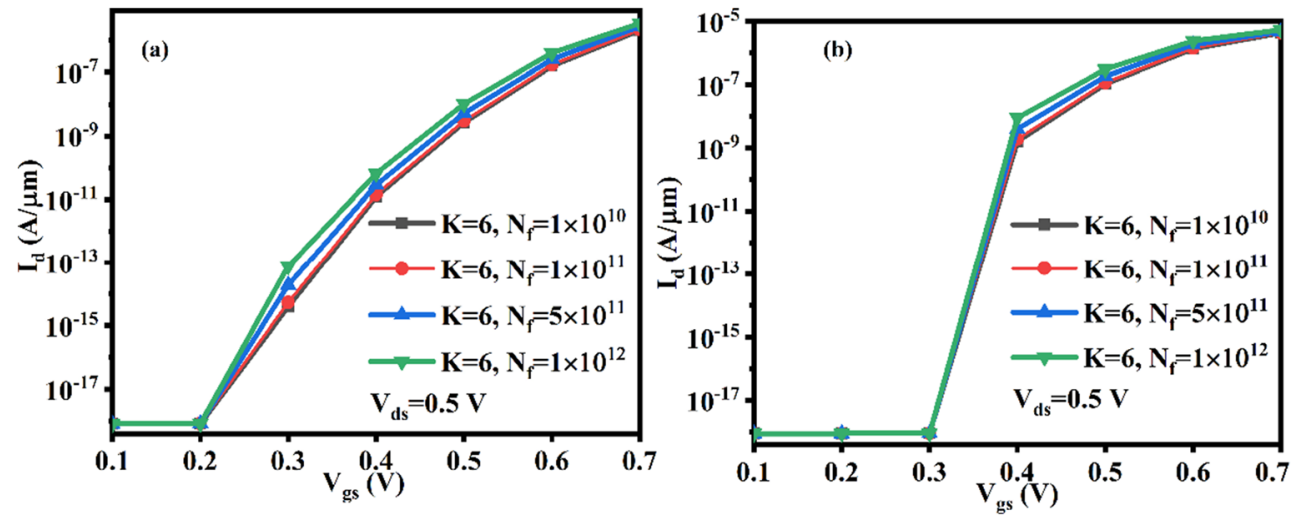
Fig. $8 I_{d} \sim V_{g s}$ characteristics of negatively charged biomolecules for $\mathbf{a} \mathrm{Ge} / \mathrm{GaAs}$ TFET biosensor $\mathbf{b}$ $\mathrm{Ge} / \mathrm{GaAs}$ NCTFET biosensor
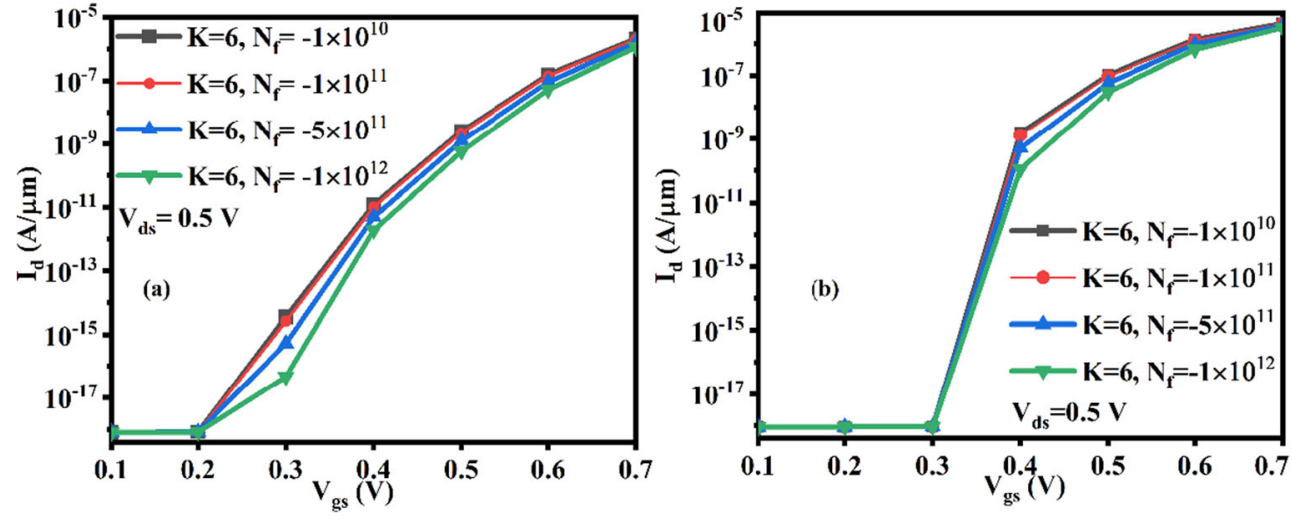

from the plot that for the whole range of $\mathrm{V}_{\mathrm{gs}}, \tau$ is lower in case of Ge/GaAs NC-TFET for better sensing capability than Ge/ GaAs TFET biosensors. A good biosensor capability is decided by its sensitivity. Greater the sensing capability, more effective would be the biomolecule identification. The drain current sensitivity is viewed as primary factor while estimating the performance of a biosensor which is defined as [34]:

Sensitivity $=\frac{I_{d}(\text { bio })-I_{d}(\text { air })}{I_{d}(\text { air })}$

In Eq. (1), the drain current in the presence and absence of biomolecules in the cavity are represented by $\mathrm{I}_{\mathrm{d}}($ bio) and $\mathrm{I}_{\mathrm{d}}$ (air), respectively. Table 2 lists the sensitivity of the proposed device for various biomolecules. As $\mathrm{K}$ increases, $\mathrm{I}_{\mathrm{ON}}$ increases with positive value of $\mathrm{V}_{\mathrm{gs}}$. In this way, sensitivity is also enhanced with increase in drain current. The increase in negative (positive) charge of biomolecules indicates the reduction (enhancement) in $\mathrm{I}_{\mathrm{ON}}$. Subsequently, the biosensor sensitivity diminishes (improves) in ON state. The most noteworthy sensitivity of the proposed biosensor is acquired at medium $V_{g}$ due to the amplification of $I_{d}$ in sub-threshold region of both the devices. Figure 10 shows sensitivity of neutral, charged and uncharged biomolecules. The highest sensitivity of the order of $10^{13}$ and $10^{7}$ for $\mathrm{K}=12$ is achieved in Ge/GaAs-NC-TFET and Ge/GaAs-TFET based biosensors, respectively due to high current achieved at low voltage in $\mathrm{Ge} /$ GaAs-NC-TFET and low SS. The low SS is due to the effect of NC (voltage amplification) in FE-DE stack, which is shown in Table 4. Similarly, $\mathrm{V}_{\text {th }}$ of Ge/GaAs-NCTFET biosensor is less as compared to Ge/GaAs-TFET biosensor. Therefore, from Table 3, we found that $\mathrm{I}_{\mathrm{ON}}$ improves by almost a decade in case of Ge/GaAs-NCTFET biosensor as compared to $\mathrm{Ge} /$ GaAs-TFET biosensor by maintaining nearly same $\mathrm{I}_{\mathrm{OFF}}$ due to capacitance matching in FE-DE region. Finally, in Table 4 we have compared the sensitivity of our proposed device with the FET based biosensors available in literature. Though there is substantial variation in dimensions and dielectric constant, still it is found that Ge/GaAs-NC-TFET provide extremely high sensitivity as compared to other devices.

\section{Conclusion}

Traditional CMOS devices are prone to short channel effects, which restricts its use in low power circuitries and open pathways for intensifying the research on novel electronic devices. TFET is one such potential device with lower SS and $\mathrm{I}_{\mathrm{OFF}}$ in comparison to that of CMOS but it suffers from low $\mathrm{I}_{\mathrm{ON}}$ and higher ambipolarity. The concept of NC coupled with III-V material based TFETs yield some interesting results, which are summarized in this paper. FE material is introduced in half
Fig. 9 Transit time $(\tau)$ of a Ge/ GaAs TFET biosensor, b Ge/ GaAs NCTFET biosensor with different dielectric constants $(\mathrm{K})$
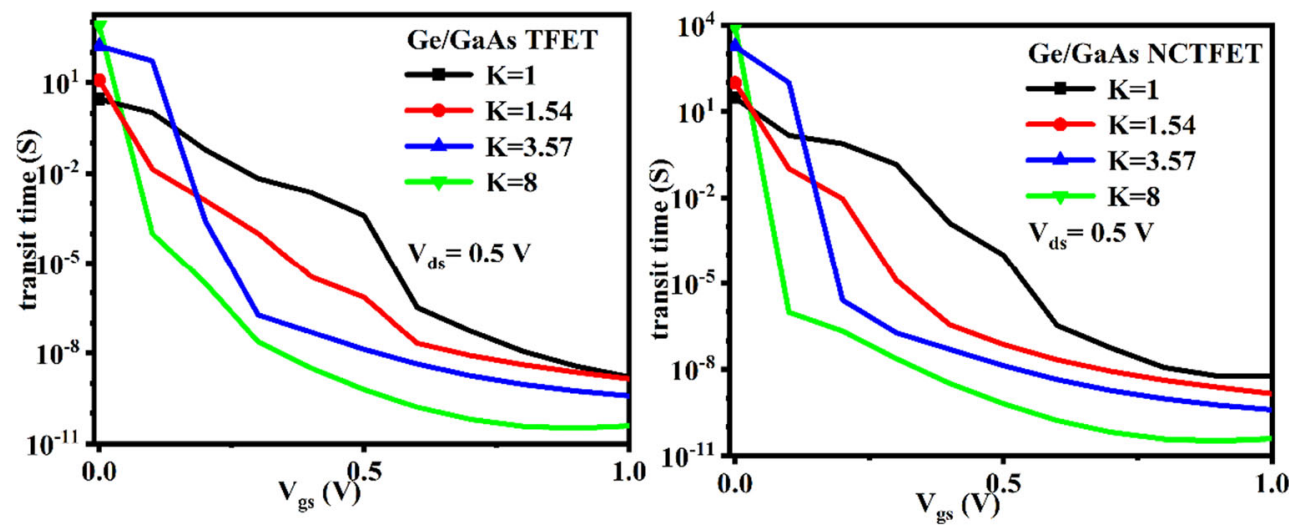
Table 2 Sensitivity of different neutral and charged biomolecules for TFET biosensor and NCTFET biosensor

Fig. 10 Sensitivity of a neutral biomolecules, b positively charged biomolecules, $\mathrm{K}=6 \mathrm{c}$ negatively charged, $\mathrm{K}=6$ biomolecules for Ge/GaAs TFET and $\mathrm{Ge} / \mathrm{GaAs}$ NCTFET biosensor at $\mathrm{V}_{\mathrm{ds}}=0.5 \mathrm{~V}$

\begin{tabular}{llll}
\hline \multirow{2}{*}{ Biomolecules } & Dielectric constants $(\mathrm{K})$ & \multicolumn{2}{c}{ Sensitivity due to ON current $\mathrm{V}_{\mathrm{gs}}=1 \mathrm{~V}, \mathrm{~V}_{\mathrm{ds}}=0.5 \mathrm{~V}$} \\
\cline { 3 - 4 } & & TFET-Bio & NCTFET-Bio \\
\hline Neutral & 1.54 & $6.25 \times 10^{4}$ & $4.12 \times 10^{6}$ \\
& 2.63 & $6.29 \times 10^{5}$ & $2.19 \times 10^{8}$ \\
& 3.57 & $6.82 \times 10^{6}$ & $1.72 \times 10^{11}$ \\
& 4.7 & $1.48 \times 10^{7}$ & $2.58 \times 10^{12}$ \\
& 6.3 & $4.04 \times 10^{7}$ & $9.08 \times 10^{12}$ \\
& 8 & $5.92 \times 10^{7}$ & $9.41 \times 10^{12}$ \\
& 12 & $6.01 \times 10^{7}$ & $9.07 \times 10^{13}$ \\
Positive Charged $\left(\mathrm{C} / \mathrm{cm}^{2}\right)$ & $\mathrm{K}=6, \mathrm{~N}_{\mathrm{f}}=1 \times 10^{10}$ & $3.57 \times 10^{7}$ & $5.24 \times 10^{12}$ \\
& $\mathrm{~K}=6, \mathrm{~N}_{\mathrm{f}}=1 \times 10^{11}$ & $3.75 \times 10^{7}$ & $5.37 \times 10^{12}$ \\
& $\mathrm{~K}=6, \mathrm{~N}_{\mathrm{f}}=5 \times 10^{11}$ & $4.65 \times 10^{7}$ & $5.92 \times 10^{12}$ \\
& $\mathrm{~K}=6, \mathrm{~N}_{\mathrm{f}}=1 \times 10^{12}$ & $5.91 \times 10^{7}$ & $6.59 \times 10^{12}$ \\
$\mathrm{~K}=6, \mathrm{~N}_{\mathrm{f}}=-1 \times 10^{10}$ & $3.53 \times 10^{7}$ & $5.22 \times 10^{12}$ \\
Negative charged $\left(\mathrm{C} / \mathrm{cm}^{2}\right)$ & $\mathrm{K}=6, \mathrm{~N}_{\mathrm{f}}=-1 \times 10^{11}$ & $3.35 \times 10^{7}$ & $5.09 \times 10^{12}$ \\
& $\mathrm{~K}=6, \mathrm{~N}_{\mathrm{f}}=-5 \times 10^{11}$ & $2.62 \times 10^{7}$ & $4.53 \times 10^{12}$ \\
$\mathrm{~K}=6, \mathrm{~N}_{\mathrm{f}}=-1 \times 10^{12}$ & $1.88 \times 10^{7}$ & $3.83 \times 10^{12}$ \\
\hline
\end{tabular}

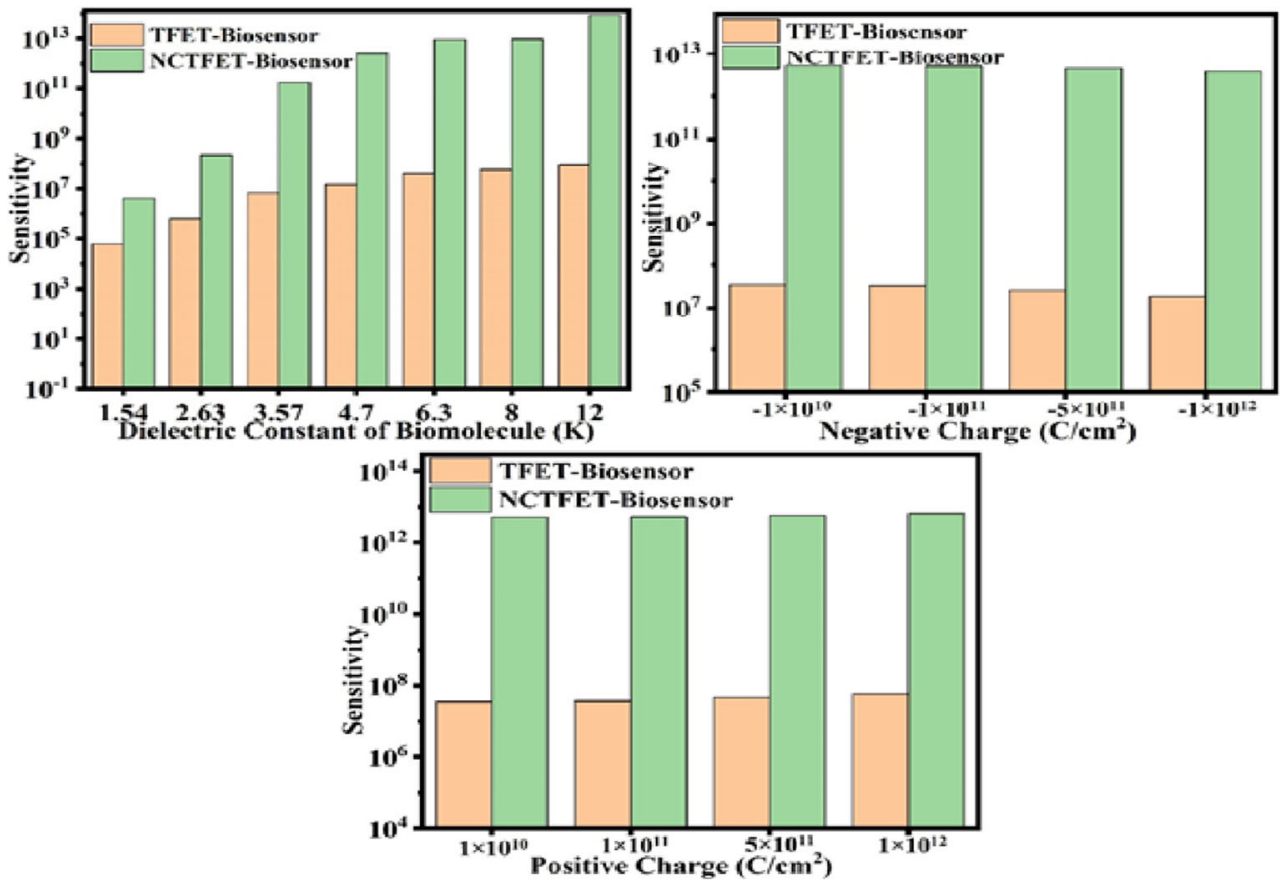

of channel length between gate electrode and oxide insulator to form a nano cavity for trapping biomolecules in the proposed TFET biosensor. NC played a big role in increasing the current values of the device. As we introduced biomolecules having different values of dielectric constant, the current and consequently the sensitivity of the biosensor increases. The key thing in NCTFET based biosensor is that we can get high ON state current by applying lesser $\mathrm{V}_{\mathrm{gs}}$, which causes steep SS and high sensitivity. Besides, introduction of FE material
Table 3 Comparison of electrical parameters between the proposed TFET biosensor and NCTFET biosensor for $\mathrm{K}=12$

\begin{tabular}{lllll}
\hline Device & $\mathrm{V}_{\text {th }}(\mathrm{V})$ & $\mathrm{SS}(\mathrm{mV} / \mathrm{dec})$ & $\mathrm{I}_{\mathrm{ON}}(\mathrm{A} / \mu m)$ & $\mathrm{I}_{\mathrm{OFF}}(\mathrm{A} / \mu m)$ \\
\hline Ge/GaAs- TFET & 0.55 & 21 & $8.34 \times 10^{-6}$ & $7.31 \times 10^{-18}$ \\
Ge/GaAs NCTFET & 0.43 & 13 & $9.56 \times 10^{-5}$ & $8.57 \times 10^{-18}$ \\
\hline
\end{tabular}


Table 4 Sensitivity comparison among various biosensors available in literature

\begin{tabular}{lll}
\hline Sl. no. & Bio-FETs & Sensitivity \\
\hline 1 & {$[17],\left(\mathrm{L}_{\text {gap }}=10 \mathrm{~nm}, \mathrm{H}_{\text {gap }}=5 \mathrm{~nm}, \mathrm{~L}_{\text {gate }}=42 \mathrm{~nm}, \mathrm{~K}=4\right)$} & $1 \times 10^{5}$ \\
2 & {$[17],\left(\mathrm{L}_{\text {gap }}=10 \mathrm{~nm}, \mathrm{H}_{\text {gap }}=5 \mathrm{~nm}, \mathrm{~L}_{\text {gate }}=20 \mathrm{~nm}, \mathrm{~K}=4\right)$} & $1 \times 10^{6}$ \\
3 & DMTFET (Full gate) $[23]$, & $2.5 \times 10^{4}$ \\
& $\left(\mathrm{~L}_{\text {gap }}=10 \mathrm{~nm}, \mathrm{H}_{\text {gap }}=5 \mathrm{~nm}, \mathrm{~L}_{\text {gate }}=42 \mathrm{~nm}, \mathrm{~K}=4\right)$ & \\
4 & DMTFET (Short gate) $[23], \mathrm{L}_{\text {gap }}=10 \mathrm{~nm}, \mathrm{H}_{\text {gap }}=5 \mathrm{~nm}, \mathrm{~L}_{\text {gate }}=42 \mathrm{~nm} \mathrm{K=4}$ & $2.5 \times 10^{5}$ \\
5 & {$[20],\left(\mathrm{L}_{\text {gap }}=10 \mathrm{~nm}, \mathrm{H}_{\text {gap }}=5 \mathrm{~nm}, \mathrm{~L}_{\text {gate }}=30 \mathrm{~nm}, \mathrm{~K}=6\right)$} & $2 \times 10^{4}$ \\
6 & {$[34]\left(\mathrm{L}_{\text {gap }}=30 \mathrm{~nm}, \mathrm{H}_{\text {gap }}=9 \mathrm{~nm}, \mathrm{~L}_{\text {gate }}=100 \mathrm{~nm}, \mathrm{~K}=10\right)$} & $3 \times 10^{6}$ \\
7 & $\mathrm{Ge} / \mathrm{GaAs}-\mathrm{TFET}\left(\mathrm{t}_{\mathrm{ox}}=2 \mathrm{~nm}, \mathrm{~L}_{\text {ch }}=20 \mathrm{~nm}, \mathrm{t}_{\mathrm{Ferro}}=5 \mathrm{~nm}, \mathrm{~K}=6\right)$ & $4.04 \times 10^{7}$ \\
8 & $\mathrm{NC}-\mathrm{Ge} / \mathrm{GaAs}-\mathrm{TFET}\left(\mathrm{t}_{\mathrm{ox}}=2 \mathrm{~nm}, \mathrm{~L}_{\text {gate }}=20 \mathrm{~nm}, \mathrm{t}_{\mathrm{Ferro}}=5 \mathrm{~nm}, \mathrm{~K}=6\right)$ & $9.08 \times 10^{12}$ \\
\hline
\end{tabular}

under the gate terminal increases the steepness of the energy bands at the source-channel junction and obviously, improvement in the $\mathrm{I}_{\mathrm{ON}}$ of the device. Higher $\mathrm{I}_{\mathrm{ON}}$ is achieved in Ge/ GaAs based NC-TFET biosensor, when compared with that of the Ge/GaAs based TFET biosensor. Additionally, improvement in the SS of the device is seen because of the steepness of subthreshold conduction current. In our proposed device, a SS of $13 \mathrm{mV} /$ decade is obtained which is very low when compared to $21 \mathrm{mV} /$ decade for the Ge/GaAs-based TFET biosensor. Indeed, from the above investigation, it is sensible to conclude that Ge/GaAs based NC-TFET biosensor is superior with respect to $\mathrm{Ge} / \mathrm{GaAs}$ based TFET biosensor in terms of sensitivity.

Code Availability Not Applicable.

Author Contributions All the authors have contributed to the design, investigation, conceptualization and formal analysis and design. Author Omdarshan Paul prepared the first draft of the manuscript after performing the simulation study and validation. Author Chithraja Rajan edited the manuscript after data analysis and validation. D P Samajdar commented on the manuscript and supervised the entire work. All the authors read and approved the final version of the manuscript. Authors Hidouri Tarek and Samia Nasr provided some fruitful discussions and comments regarding the content of the work.

Funding No funding was received for this research work.

Data Availability Not applicable.

\section{Declarations}

Consent to Participate All the authors contributed voluntarily to this work.

Consent for Publication In accordance with the copyright transfer or open access rules.

Acknowledgements and Ethics Approval Not Applicable.

Conflict of Interest The authors declare that they have no conflict of interest.

\section{References}

1. Bohk-Ewald C, Dudel C, Myrskylä M (2020). Int J Epidemiol 49: 1963-1971

2. Acquah C, Danquah MK, Agyei D, Moy CK, Sidhu A, Ongkudon CM (2016). Crit Rev Biotechnol 36:1010-1022

3. Caygill RL, Blair GE, Millner PA (2010). Anal Chim Acta 681:815

4. Cesewski E, Johnson BN (2020). Biosens Bioelectron 159:112214

5. Dak P, Ebrahimi A, Swaminathan V, Duarte-Guevara C, Bashir R, Alam MA (2016). Biosensors 6(2):14

6. Chan JFW, Yuan S, Kok KH, To KKW, Chu H, Yang J, Xing F, Liu J, Yip CCY, Poon RWS, Tsoi HW (2020). Lancet 395:514 523

7. Kim CH, Jung C, Park HG, Choi YK (2008). Biochip J 2:127-134

8. Gautam R, Saxena M, Gupta RS, Gupta M (2012) Numerical Model of Gate-All-Around MOSFET With Vacuum Gate Dielectric for Biomolecule Detection. IEEE Electron Device Lett 33:1756-1758

9. Kannan N, Kumar MJ (2013) Dielectric-Modulated ImpactIonization MOS Transistor as a Label-Free Biosensor. IEEE Electron Device Lett 34:1575-1577

10. Choi JM, Han JW, Choi SJ, Choi YK (2010). IEEE Trans Electron Devices 57:3477-3484

11. Singh D, Pandey S, Nigam K, Sharma D, Yadav DS, Kondekar P (2017). IEEE Trans Electron Devices 64:271-278

12. Agnihotri SK, Samajdar DP, Rajan C, Yadav AS, Gnanesh G (2021) Performance analysis of gate engineered dielectrically modulated TFET biosensors. Int J Electron 108:607-622

13. Acharya B, Mishra GP (2020) Design and Analysis of Dual-MetalGate Double-Cavity Charge-Plasma-TFET as a Label Free Biosensor. IEEE Sensors J 20:13969-13975

14. Venkata B et al (2017) Single image defogging based on particle swarm optimization. Micro Nano Lett 13:452-456

15. Dixit A, Samajdar DP, Chauhan V (2021). IEEE Trans Electron Devices. https://doi.org/10.1109/TED.2021.3107368

16. Dixit A, Samajdar DP, Bagga N (2021). Semicond Sci Technol 36: 13

17. Narang R, Reddy KS, Saxena M, Gupta RS, Gupta M (2012). IEEE Trans Electron Devices 59:2809-2817

18. Chandar DB, Vadthiya N, Kumar A, Mishra RA (2013) Suppression of Short Channel Effects(SCEs) by Dual Material Gate Vertical Surrounding Gate(DMGVSG) MOSFET: 3-D TCAD Simulation. Proc Eng 64:125-132

19. Chaudhry A, Kumar MJ (2004). IEEE Trans Device Mater Reliab 4:99-109

20. Wangkheirakpam VD, Bhowmick B, Pukhrambam PD (2020) N+ Pocket Doped Vertical TFET Based Dielectric-Modulated 
Biosensor Considering Non-Ideal Hybridization Issue: A Simulation Study. IEEE Trans Nanotechnol 19:156-162

21. Yadav S, Rajan C, Sharma D, Balotiya S (2019) International symposium on VLSI design and test. Springer, Singapore, pp 694-701

22. Rajan C, Samajdar DP, Patel J, Lodhi A, Agnihotri SK, Sharma D, Kumar A (2020) Linearity and Reliability Analysis of an Electrically Doped Hetero Material Nanowire TFET. J Electron Mater 49:4307-4317

23. Kanungo S, Chattopadhyay S, Gupta PS, Rahaman H (2015) Comparative Performance Analysis of the Dielectrically Modulated Full- Gate and Short-Gate Tunnel FET-Based Biosensors. IEEE Trans Electron Devices 62:994-1001

24. Salahuddin S, Datta S (2008). Nano Lett 8:405-410

25. Khan AI, Yeung CW, Hu C, Salahuddin S (2011) 2011 international Electron devices meeting. IEEE, pp 11-13

26. Zhao Y, Liang Z, Huang Q, Chen C, Yang M, Sun Z, Zhu K, Wang H, Liu S, Liu T, Peng Y (2019) A Novel Negative Capacitance Tunnel FET With Improved Subthreshold Swing and Nearly Non-Hysteresis Through Hybrid Modulation. IEEE Electron Device Lett 40:989-992

27. Rajan C, Paul O, Samajdar D.P. et al. Performance Analysis of IIIV and IV Semiconductors Based Double Gate Hetero Material Negative Capacitance TFET. Silicon (2022). https://doi.org/10. 1007/s12633-022-01667-x
28. Silvaco ATLAS (2013) Device simulation software. Silvaco Int, Santa Clara, CA

29. Kwon D, Cheema S, Shanker N, Chatterjee K, Liao YH, Tan AJ, Hu C, Salahuddin S (2019) Negative Capacitance FET With 1.8nm-Thick Zr-Doped HfO2Oxide. IEEE Electron Device Lett 40: 993-996

30. Gandhi R, Chen Z, Singh N, Banerjee K, Lee S (2011). IEEE Electron Device Lett 32:437-439

31. Müller J, Polakowski P, Mueller S, Mikolajick T (2015). ECS J Solid State SciTechnol 4:30-35

32. Toprasertponga K, Tahara K, Takenaka M, Takagi S (2020). Appl Phys Lett 116. https://doi.org/10.1063/5.0008060

33. Lodhi A, Rajan C, Behera AK, Samajdar DP, Soni D, Yadav DS (2020) Sensitivity and sensing speed analysis of extended nanocavity and source over electrode in $\mathrm{Si} / \mathrm{SiGe}$ based TFET biosensor. Appl Phys A 126:1-8

34. Narang R, Saxena M, Gupta M (2015) Comparative Analysis of Dielectric-Modulated FET and TFET-Based Biosensor. IEEE Trans Nanotechnol 14:427-435

Publisher's Note Springer Nature remains neutral with regard to jurisdictional claims in published maps and institutional affiliations. 